\section{Locus of selectivity in a visual memory task using a color value indicator}

\author{
DAVID W. MARTIN and EILEEN RICHARDS* \\ New Mexico State University, Las Cruces, New Mexico 88001
}

Selection of high vs low valued letters from a briefly presented visual array was investigated. An indicator specifying the value of letters occurred before or after the array. Selection could occur during input to memory or during output from memory in the before condition, but only at output in the after condition. Previous research using an alphabetic instruction, which required letter identification prior to selection, attributed selection to output. A color instruction in the present experiment, not requiring letter identification, showed the locus of selection to be at input. Experiment 2 replicated these findings and used an interference technique to add further support. In addition, contrary to previous findings, these results indicated that the selection process caused no deficit in overall recall.

Tolkmitt and Christ (1970) briefly presented arrays containing eight letters. In differential payoff groups, half of these letters (high) were worth 2,4 , or 8 points each compared to 1-point (low) letters. In equal value groups, each letter was worth 1 point. Either prior to (before) or following (after) the array, the high value letters were indicated. In the before condition, S could have selected and retained high value letters from the array prior to selecting low value letters. In either the before or after condition, a second type of selection was possible. The $S$ could have retained all of the letters regardless of value and at recall selected the high value letters first. While Tolkmitt and Christ called these processes "selective retention" and "selective recall," respectively, in this paper they will be called selection at input to memory or at output from memory.

Since these investigators reported more high than low value letters recalled in both the before and after conditions, selection was attributed primarily to output. In addition, poorer overall recall of letters was found in the before condition with differential payoff compared to the equal value group. This indicated that attempts to select at input loaded Ss' processing capacity and reduced the overall amount of information available for subsequent recall.

In a second experiment, exposure time of the letter array was set at 0.5 or $2.5 \mathrm{sec}$. More letters were recalled at the longer exposure. In addition, the differences in recall between high value and low value letters were

*Now at Columbia State School, Columbia, Louisiana 71418. Requests for reprints should be sent to David $W$. Martin Department of Psychology, New Mexico State University, Las Cruces, New Mexico 88001 . greater for longer exposures. These differences also tended to be greater for the after condition than for the before with the 0.5 -sec exposure, but the reverse was true with the 2.5 -sec exposure.

The experiments reported here are attempts to determine if Tolkmitt and Christ's results were dependent upon the type of selection indicator used in the experimental task. Their Ss observed "A-J" or "Q-Z" immediately before or after presentation of the letter array. Processing of this value indicator is quite a complex task. Other ways of indicating the high value letters are, of course, possible. While little research has been conducted on the type of indicator used for selective attention when the array is available for 1 sec or more, much effort has been devoted to specifying the parameters of selective attention indicators when selection is from iconic memory (Neisser, 1967).

Sperling (1960), in his sixth experiment, found that selection was almost totally absent when the items to be selected were numbers only or letters only from an array containing both. He had found good selection when the indicator specified a particular row. Apparently, selection by category took so much time that the icon faded prior to completion. Eriksen and Collins (1969) and Averbach and Coriell (1961) have estimated that apprehension of even a single simple indicator and location of the display position may take as long as 200-300 msec. The selection task was even more difficult when an Arabic numeral was used to indicate the location on the display which was to be reported. These studies required selection of only one item from the required selection of four items, it could be expected that this selection would take even more time, although display. Since Tolkmitt and Christ
Eriksen and Colegate (1971) and Allport (1971) present data indicating that selection time for multiple stimuli is not an additive function of selection time for an individual stimulus.

von Wright (1968) provides additional evidence that the type of indicator used for selective attention determines how much time the process takes. He found good selection when the indicator was color, location, achromatic color, or size. However, when the letters to be reported were to be selected based on a tilt of 0 or $45 \mathrm{deg}$, selection was quite poor. An explanation for this result was given; perhaps letters had to be ittentined before orientation could be determined.

The data reported above, taken in sum, indicate that the process of selective attention takes time. Even for a selection buted upon a simple physical dimemsion, this time is substantial (200-300 msec). When the selection requires complete identification of items, the selection time becomes even longer. Selective attention in the Tolkmitt and Christ experiment required item identification and also comparison of the identified item with a large memory set (first or last 10 letters of the alphabet). It is quite possible that this selection process would take so long that only one or two items could be selected during the 1 sec the array was present. For this reason, $S$ may have adopted a strategy in which at input the items were stored without regard for value, while at output high value items were selected for recall prior to low value items. This strategy would produce the results found by Tolkmitt and Christ; selection would occur primarily at output.

Quite a different strategy might be optimal if the selection were based upon a physical dimension and did not require letter identification. Color was chosen in the present experiment, since several investigators had found it to be an indicator which required a minimal amount of processing time (Allport, 1971; von Wright, 1968). The optimal strategy in the present experiment would involve selecting high value letters based upon color information, then identifying and retaining these. Only after storing all of the high value letters would $S$ attempt to identify and retain low value letters. Thus, when selective attention can occur without letter identification, it may occur during input.

Several predictions are offered. First, since the processing load of selection may be reduced, the differential payoff group's overall performance should more approximate 


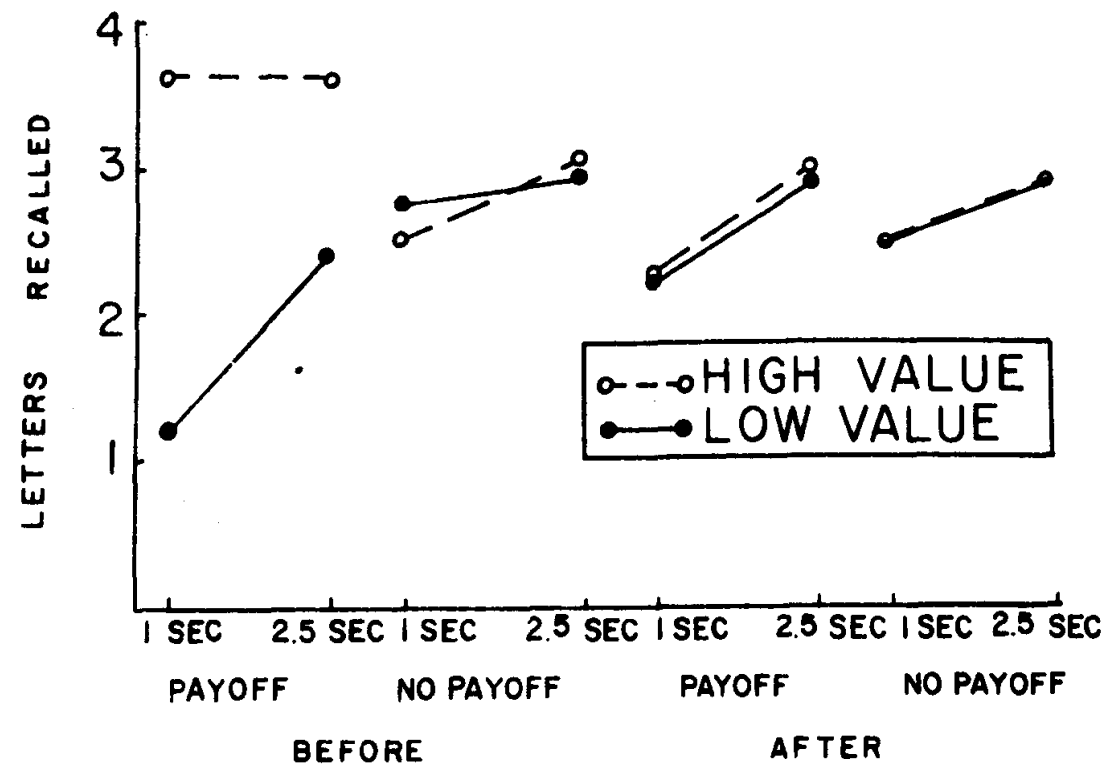

Fig. 1. The mean number of high and low value letters correctly recalled in Experiment 1 as a function of before-after, payoff/no payoff, and exposure time.

the equal value group in all conditions. Second, since the selection task may take less time, a greater degree of selection should be expected than found by the previous investigators. Third, since the high value letters may be selected without first identifying the letter itself, selection will occur primarily during input rather than during output.

\section{EXPERIMENT 1 \\ Procedure}

Eight 48-pt letters in a $5.1 \times 10.2 \mathrm{~cm}$ array were presented in two rows of four letters on each of 40 white $10.2 \times 12.7 \mathrm{~cm}$ cards. Four of the letters were black and four were blue, these colors being easily discriminable. The letters were chosen randomly from the alphabet, were assigned randomly one of the two colors, and were assigned randomly a position on the card, with the restriction that each letter appear only once on a card. The value indicator cards consisted of 2 white $10.2 \times 12.7 \mathrm{~cm}$ cards with either a black or a blue 2-mm-wide border enclosing a $6 \times 6 \mathrm{~cm}$ square. The letter arrays and borders were presented for either 1 or $2.5 \mathrm{sec}$ by means of a two-channel tachistoscope (Scientific Prototype, Model 800-F).

Forty student volunteers from introductory psychology classes at New Mexico State University served as Ss and were assigned randomly to four groups of $10 \mathrm{Ss}$ each. The Ss were run individually, each serving for approximately $40 \mathrm{~min}$.

The design was a $2^{4}$ factorial, with exposure time and payoff/no payoff as
between-Ss effects and before-after and high-low as within-Ss effects. The exposure times were 1.0 and $2.5 \mathrm{sec}$. Before-after refers to the indication of letter value preceding or following the array, and high-low refers to the 4-point or 1-point value of the letters in the array. Each $S$ received an equal number of before and after trials, and each color indicator was used an equal number of times and was distributed randomly over trials.

The E informed the payoff groups that they would be competing for a $\$ 5$ prize by accumulating the greatest number of points, that some letters were worth four times other letters, and that high value would be indicated by the color border preceding or following the array. The no-payoff groups were told to ignore color and simply to write down as many letters as passible. The $S$ then received 4 practice trials prior to the 40 experimental trials.

In the before condition, following a verbal warning, the tachistoscope presented a 1-sec exposure of the indicator preceding a 1-sec exposure of the letter array. (In the 2.5 -sec groups, the indicator and letters were exposed for 2.5 sec each.) After the stimulus presentation, $S$ was given $30 \mathrm{sec}$ to write down the letters he could recall. The $\mathbf{S}$ was then instructed to look into the tachistoscope for the next trial. The same procedure was followed in the after condition, except that the indicator followed the array.

\section{Results}

An analysis of variance was conducted on the number of letters correctly recalled in each condition. Main effects reaching significance included exposure time $[F(1,36)=$ $17.0, \mathrm{p}<.01]$, high-low $[\mathrm{F}(1,36)=$ 84.7, $p<.01]$, and before-after $[F(1,36)=35.5, p<.01]$. The significant interactions can be seen in Fig. 1. In the before-payoff groups, at both 1 and $2.5 \mathrm{sec}$, the number of high value letters recalled was significantly greater than low value $[F(1,36)=$ $11.8, p<.01]$. This difference was not found in any after condition or in any no-pay off condition. The High-Low by Exposure Time interaction $[F(1,36)=4.5, p<.05]$ also indicates that for the before-payoff condition the difference between the high value letter recall and low value recall was greater at $1 \mathrm{sec}$ than at 2.5 sec.

Each of the predictions was supported. Tolkmitt and Christ found the overall level of performance for the $1: 1$ payoff group to be superior to the $2: 1,4: 1$, or $8: 1$ payoff groups in the before condition. In contrast, no overall performance differences were found between payoff conditions in the present results. The selection task was apparently done so quickly and efficiently that it caused no interference with overall performance.

The second prediction was also supported. The previous investigators in the 1-sec exposure experiment found more high value letters recalled than low value letters in all differential payoff conditions. The sizes of these differences in letters recalled were: before-2:1 = .04, before-4:1 $=.29$, before- $8: 1=.08$, after- $2: 1=.10$, after-4:1 $=.12$, and after-8:1 $=.03$. In the present experiment, for the 1 -sec condition there was no selection with the after instruction. The degree of selection in the before condition was much higher than that previousiy found-2.45 letters.

The third prediction concerned the role of input vs output in the selection process. Tolkmitt and Christ attributed their results primarily to selection at output. The results reported here, however, support the role of input. No differences were found between recall of high and low value letters in any of the after conditions. Large differences were found in the before conditions when differential payoff was given. It would seem, then, that the degree to which input vs output operate in the selection of letters from an array depends upon the time required for selective attention to take place. A color indicator takes much less time to process than does an alphabetic indicator.

In the before condition, a greater 
degree of selection was found for $1 \mathrm{sec}$ than for $2.5 \mathrm{sec}$. This finding seems a bit surprising. The results in Fig. 1 , however, show why this is the case. The number of high value letters recalled for 1 - and $2.5 \mathrm{sec}$ payoffs was exactly the same-3.62. This figure approaches the ceiling of 4.0 and indicates very good recall of high value letters. The major difference in the exposure time conditions concerns low value letters. More of these were recalled at $2.5 \mathrm{sec}$ than at $1 \mathrm{sec}, 1.17$ $v 2.40$. It can be seen, then, that $S$ must first have retained nearly all of the high value letters and then used the time left to retain as many of the low value letters as possible.

\section{EXPERIMENT 2}

Experiment 1 indicated that when a color dimension was used as an indicator for selective attention, the high value letters were recalled better than the low value letters primarily due to selection at input. Experiment 1 was an attempt to replicate this result and, in addition, to provide further support for the role of input using an interference technique. The interference was produced by the use of a counterindicator. As in Experiment 1, a colored border occurring before or after the array indicated the color of the high valued letters. Following the trial, however, this indicator was sometimes countermanded, using another color indicator. In the before condition, if selection occurs at input, this selection would take place prior to the counterindicator. In fact, since most of the low value letters would never be retained, it would be impossible to recall them if the counterindicator reverses the original indicator. However, in the after condition, only output selection can take place. The results of Experiment 1 indicated that selection was not, in fact, occurring in the after condition. If no selection takes place, then an indicator which reverses the after indicator should in no way interfere with performance. Thus, it can be predicted that a counterindicator that reverses the original indicator will be extremely difficult for $S_{s}$ in the before condition to follow; they will not be able to use this counterindicator and will, in fact, recall more of the letters which were of high value originally but are low value following the counterindicator. In the after condition, since the results of Experiment 1 indicate that no selection took place, this counterindicator will have no effect.

Experiment 2 also corrected for a confounding variable found in both Tolkmitt and Christ and in Experiment 1. Since $S$ can respond immediately upon termination of the array in the before condition but must wait until the indicator in the after condition, time between the array and response is completely confounded with before-after. To avoid this in Experiment 2, the counterindicator always followed termination of the array by $1 \mathrm{sec}$ regardless of the presence or absence of an afterindicator. Hence, recall followed termination of the array by $2 \mathrm{sec}$ in all conditions.

\section{Procedure}

The letter arrays and indicator cards were the same as those used in Experiment 1. The same or reversed counterindicator consisted of either a blue or a black piece of tape, each separately illuminated by a CM 51 light mounted inside the tachistoscope. The tape could be seen by slight upward eye movement. The lighted areas did not obstruct the viewing channels.

The same two-channel tachistoscope used in Experiment 1 was externally controlled by a switch that also started a Hunter timer (Model C). The arrays and borders were presented by the t a chistoscope, and the counterindicator was initiated by the timer.

The Ss were obtained as in Experiment 1 and assigned randomly to two groups of $10 \mathrm{Ss}$ each.

The design was the same as in Experiment 1, with the following exceptions: The exposure time condition was fixed at 1 sec. Four of the 20 trials in each of the before and after conditions received a counterindicator which reversed the original instruction. In all other trials, the counterindicator was the same as the original indicator. The ratio of $4: 1$ for same vs reversed counterindicator trials was chosen so that $\mathbf{S}$ would not ignore the original indicator. Had the ratio been $1: 1$, then on half of the trials the original indicator would have been wrong. The $S$ could have logically ignored the original indicator on all trials.

The procedure was the same as in Experiment 1, with the exception that Ss in the payoff group were informed that the tape color lighted just before recall would be different from the earlier indicator on a few trials. The no-payoff group was told to ignore color and simply record as many letters as possible.

The before condition consisted of a $1-\mathrm{sec}$ exposure of the indicator, a $1-\mathrm{sec}$ exposure of the letter array, a 1 -sec interval, a 1 -sec exposure of lighted tape, and 30 sec of recall. The after condition consisted of a 1-sec exposure of the letter array, a $1-\mathrm{sec}$ exposure of the indicator, a $1-\mathrm{sec}$ exposure of the lighted tape, and
$30 \mathrm{sec}$ of recall. Each session lasted approximately $45 \mathrm{~min}$.

\section{Results}

An analysis of variance was conducted on the number of words correctly recalled in each condition. The data consisted of the 4 trials from the before and after conditions in which the counterindicator reversed the original indicator and 4 randomly selected trials from the 16 in which the counterindicator was the same as the original indicator.

Figure 2 illustrates the mean number of letters recalled as a function of the four conditions and payoff/no payoff. It is important to note that the value labeling on Fig. 2 refers to the value specified by the counterindicator. Thus, in the reversed condition, in which more low than high value letters were recalled, the correct interpretation is that $\mathrm{Ss}$ recalled more of what were specified as low value letters by the counterindicator. The only main effect reaching significance was conditions $[F(3,54)=11.1, p<.01]$. The four levels of conditions refers to the counterindicator being the same as, or reversed from, the before or after indicator. The only interaction reaching significance was Conditions by Payof $f / N o$ Pay off. A Newman-Keuls test was performed on the means of the four conditions. The means which were found to be different from each other at $p<.05$ were: before-same-high > before-s a me-low and before-reversed-high< before-reversed-low.

\section{Discussion}

Disregarding the results of the reversed counterindicator for the present, Experiment 2 replicates some of the important features of Experiment 1. Again, large differences were found for high vs low valued letters in the before-payoff condition, while no differences were found in the af ter-payoff condition. The total number of letters recalled for the before-payoff condition was again found to be equivalent to the before/no-payoff condition, indicating that selection at input did not degrade overall performance.

The data for the reversed condition also support an input interpretation. As predicted, $S 8$ in the before-payoff condition could not alter the original selection that had occurred. They recalled significantly more of what were originally indicated as high value letters but were countermanded into low value letters. In contrast, since very little selection had taken place in the after-payoff condition, the reversed condition had little effect on 


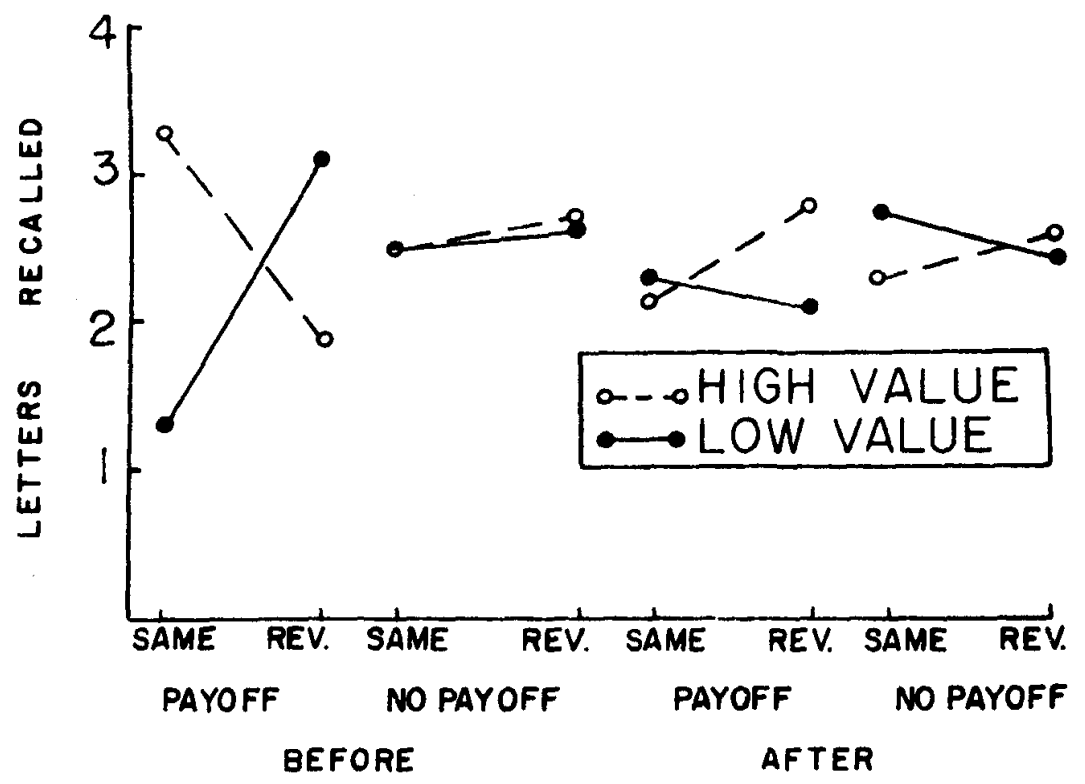

Fig. 2. The number of letters correctly recalled in Experiment 2 as a function of before-after, payoff/no payoff, and same-reversed. High and low refers to value specified by the counterindicator.

recall. In fact, a tendency existed for the after-payoff Ss to recall more high valued letters than low valued letters for the reversed condition, although this difference was nonsignificant.

\section{CONCLUSIONS}

The results of these experiments support Brown (1960), Taub (1965), Christ (1969), and Tolkmitt and Christ (1970) by showing that when differential values are assigned to items within a visual array, more higher valued items will be recalled from the array than low valued items. These letter recall when selection was required. The decrease in performance found in previous research was apparently a function of the complexity and amount of time required by the selection task.

\section{REFERENCES}

ALLPORT, D. A. Parallel encoding within and between elementary stimulus dimensions. Perception \& Psychophysics, $1971,10,104-108$

AVERBACH, E., \& CORIELL, A. S. Short-term memory in vision. Bell Sy stem Technical Journal, 1961, 40, 309-328.

BROWN, J. Evidence for a selection process d uring perception of tachistoscopically-presented stimuli. Journal of Experimental Psychology, $1960,59,176-181$.

CHRIST $R$ E. Effects of payoff and probability on recall of multisy mbol displays. Journal of General Psychology, 1969, 80, 81-92.

ERIKSEN, C. W. \& COLEGATE, R. L. Selective attention and serial processing in briefly presented visual displays. Perception \& Psychophysics, 1971, 10. 321-326.

ERIKSEN, C. W. \& COLLINS, J. F. Temporal course of selective attention. Temporal course of selective attention. Journal of Exper.

NEISSER, U. Cognitive psychology. New York: Appleton-Century-Crofts, 1967.

SPERLING, G. The in formation available in brief visual presentations. Psychological Monographs, 1960, 74(11, Whole No. 498 ).

results, however, extend the findings of Tolkmitt and Christ to a task in which selection could occur on a simple physical dimension, color. As would be predicted from research investigating the time course of selective attention from iconic storage (Eriksen \& Colegate, 1971 ; von Wright, 1968), it was found that selective attention based on color was quite different from that based on alphabetical category. With color, the selection took place at input rather than at output. In addition, these results showed no decrease in total
TAUB, H. A. Effects of differential value on recall of visual symbols. Journal of Experimental Psychology, 1965, 69. 135-143.

TOLKMITT, F., \& CHRIST, R. Differential payoff and locus of selectivity in a visual memory task. Perception \& Psychophysics, 1970, 7, 47-50.

von WRIGHT, J $M$, Selection in visual immediate memory. Quarterly Journal of Experimental Psychology, 1968, 20, 62-68.

(Accepted for publication January 31, 1972.) 